\title{
Semiconductor nanocrystals as rare-earth alternatives
}

To the Editor - As pointed out in the editorial of the January 2011 issue of Nature Photonics ${ }^{1}$, an important example of the photonics industry's dependence on rare-earth elements is the colourconvertor phosphors found in white LEDs. Phosphors containing cerium, europium and terbium, for example, are widely used as wavelength-upconverting coatings on blue LED chips to generate broadband photoluminescence spectra in the visible spectrum, peaking at yellow, green or red wavelengths, depending on the rare-earth ions used in the phosphors. Combining emission colours results in white light, making LEDs a valuable and increasingly popular form of artificial lighting for a market that reached US $\$ 10.09$ billion in 2010 and is estimated at US $\$ 46.05$ billion for $2020^{2}$.

Serious issues regarding the supply of rare-earths, as highlighted in the editorial ${ }^{1}$, could lead to disastrous effects in the solidstate lighting and backlighting industries. Fortunately, there exists a potential replacement for the rare-earth phosphors: fluorescent colloidal nanocrystal quantum dots (NQDs), formed from semiconductor nanocrystals synthesized in solution. Owing to their solution processability, stability, unique optical properties and ease of synthesis, NQDs are seen as a potentially attractive substitute for phosphors in white LEDs if large-scale, cost-effective and environmentally friendly production can be achieved.

The most favourable optical property of NQDs is their relatively narrow emission bands $(25-60 \mathrm{~nm})$, which enable colour purity and precise tunability of peak emission wavelengths (to within a few nanometres) across the entire visible range simply by using different material systems to control the size of the nanocrystals. Moreover, the wide absorption bands of NQDs makes them compatible with optical pumping when integrated onto short-wavelength LED chips. Indeed, the realization of efficient, high-quality colourconversion LEDs based on NQDs is looking promising, as shown using photometric models ${ }^{3}$ and recent prototypes of working devices $^{4-7}$. The shift from rare-earth

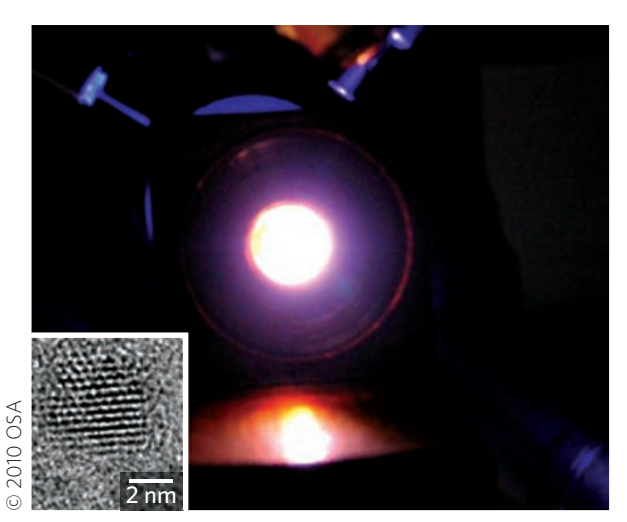

Figure 1| Photograph of a photometrically efficient NQD-integrated white LED exhibiting CRI = 89.2, LER $=357 \mathrm{Im} \mathrm{W}_{\mathrm{opt}^{-1}}$ and $\mathrm{CCT}=2,982 \mathrm{~K}$. Inset, a transmission electron microscope image of an exemplary NQD?

broadband phosphors to narrowband NQDs provides the opportunity to design LED colour-conversion coatings with high photometric performances ${ }^{3,4}$.

Although traditional rare-earth phosphor powders are able to generate a visually 'warm' white spectrum with a high colour rendering index (CRI), simultaneously accomplishing a high luminous efficacy of optical radiation (LER) remains a challenge. Conventional phosphor-based white LEDs have so far reached $274 \mathrm{~lm} \mathrm{~W}_{\text {opt }^{-1}}$ (ref. 8). Obtaining high photometric performance at high CRI, high LER and low correlated colour temperature (CCT, producing the characteristic 'warm' white spectrum) requires NQDs to be carefully designed (Fig. 1). By testing more than 200 million designs, it has been found that only around $0.001 \%$ of these can simultaneously achieve CRI $>90$ and LER $>380 \mathrm{~lm} \mathrm{~W}_{\text {opt }}{ }^{-1}$ at CCTs below 4,000 K (ref. 3). A proof-of-concept experimental demonstration of these photometrically efficient LEDs has been realized $^{4}$, together with demonstrations of long device operating times and compatibility with silicone packaging ${ }^{5,6}$. By integrating CdSe/ZnS core-shell NQDs of three different sizes (corresponding to green, yellow and red wavelengths) with a blue-emitting InGaN/GaN LED, an impressive photometric performance with an LER of $357 \mathrm{~lm} \mathrm{~W}_{\mathrm{opt}^{-1}}$, a CRI of 89.2 and a CCT of $2,982 \mathrm{~K}$ has recently been achieved ${ }^{7}$.

In summary, the supply issues of rareearth resources raise a threat to the lighting industry, with rare-earth phosphors having a stranglehold over artificial lighting. There is therefore a strong need to develop NQD-based colour converters by funding research and investing into this area. NQDs possess important capabilities and offer unique benefits for use in solidstate lighting, as well as providing new opportunities for designing warm-white, high-CRI and high-LER coatings. Although examples of state-of-the-art demonstrations are very promising, there are still important challenges that must be addressed. Efficient NQDs contain cadmium, which makes them unsuitable for use in environment friendly devices. Providing cadmium-free NQDs in amounts sufficient for the massproduction of colour-conversion LEDs is another area in which further research and development effort is needed.

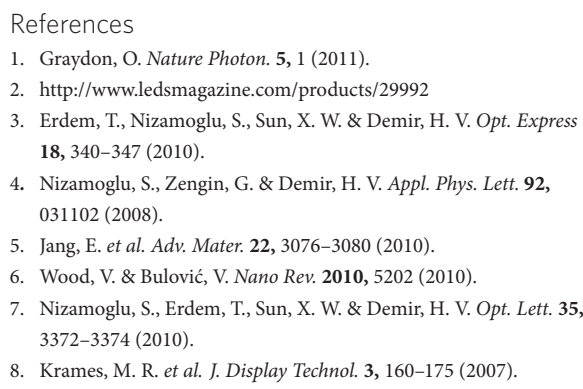

Talha Erdem ${ }^{1}$ and Hilmi Volkan Demir ${ }^{1,2 \star}$ 'Department of Electrical and Electronics Engineering, Department of Physics and UNAM - Institute of Materials Science and Nanotechnology, Bilkent University, Ankara 06800, Turkey. ${ }^{2}$ Luminous! Semiconductor Lighting and Displays Center of Excellence, School of Electrical and Electronic Engineering, School of Physical and Mathematical Sciences, Physics and Applied Physics Division, Nanyang Technological University, 50 Nanyang Avenue, 639798 Singapore. *e-mail: hvdemir@ntu.edu.sg; volkan@bilkent.edu.tr 